\title{
BMJ Open Managing cognitive impairment following stroke: protocol for a systematic review of non-randomised controlled studies of psychological interventions
}

\author{
Niamh A Merriman, ${ }^{1}$ Eithne Sexton, ${ }^{1}$ Nora-Ann Donnelly, ${ }^{2}$ Grainne McCabe, ${ }^{3}$ \\ Mary E Walsh, ${ }^{1,4}$ Daniela Rohde, ${ }^{1}$ Ashleigh Gorman, ${ }^{1}$ Isabelle Jeffares, ${ }^{1}$ \\ Niall Pender, ${ }^{5}$ David Williams, ${ }^{6,7}$ Frances Horgan, ${ }^{4}$ Frank Doyle, ${ }^{1}$ Maev-Ann Wren, ${ }^{2}$ \\ Kathleen E Bennett, ${ }^{1}$ Anne Hickey ${ }^{1}$
}

To cite: Merriman NA, Sexton E, Donnelly N-A, et al. Managing cognitive impairment following stroke: protocol for a systematic review of non-randomised controlled studies of psychological interventions. BMJ Open 2018;8:e019001. doi:10.1136/ bmjopen-2017-019001

- Prepublication history and additional material for this paper are available online. To view these files, please visit the journal online (http://dx.doi. org/10.1136/bmjopen-2017019001).

Received 10 August 2017 Revised 13 October 2017 Accepted 17 November 2017

CrossMark

For numbered affiliations see end of article.

Correspondence to Dr Niamh A Merriman; niamhmerriman@rcsi.ie

\section{ABSTRACT}

Introduction Stroke is one of the primary causes of death and disability worldwide, leaving a considerable proportion of survivors with persistent cognitive and functional deficits. Despite the prevalence of poststroke cognitive impairment, there is no established treatment aimed at improving cognitive function following a stroke. Therefore, the aims of this systematic review are to identify psychological interventions intended to improve poststroke cognitive function and establish their efficacy.

Methods and analysis A systematic review of nonrandomised controlled studies that investigated the efficacy of psychological interventions aimed at improving cognitive function in stroke survivors will be conducted. Electronic searches will be performed in the PubMed, Embase and PsycINF0 databases, the search dating from the beginning of the index to February 2017. Reference lists of all identified relevant articles will be reviewed to identify additional studies not previously identified by the electronic search. Potential grey literature will be reviewed using Google Scholar. Titles and abstracts will be assessed for eligibility by one reviewer, with a random sample of $50 \%$ independently double-screened by second reviewers. Any discrepancies will be resolved through discussion, with referral to a third reviewer where necessary. Risk of bias will be assessed with the Risk of Bias in Nonrandomized Studies of Interventions tool. Meta-analyses will be performed if studies are sufficiently homogeneous. This review will follow the Preferred Reporting Items for Systematic Reviews and Meta-Analyses statement. The quality of the evidence regarding cognitive function will be assessed according to the Grading of Recommendations Assessment, Development and Evaluation.

Ethics and dissemination This systematic review will collect secondary data only and as such ethical approval is not required. Findings will be disseminated through presentations and peer-reviewed publication. This review will provide information on the effectiveness of psychological interventions for poststroke cognitive impairment, identifying which psychological interventions are effective for improving poststroke cognitive function.
Strengths and limitations of this study

- This systematic review protocol will be reported in accordance with the Preferred Reporting Items for Systematic Reviews and Meta-Analyses statement.

- Three databases covering the medical and psychological peer-reviewed literature will be searched.

- The quality of the evidence will be assessed based on the Grading of Recommendations Assessment, Development and Evaluation.

- This systematic review will not include interventions based on pharmacological or non-psychological treatments and will include stroke patients only.

PROSPERO registration number CRD42017069714.

\section{INTRODUCTION}

Stroke is one of the primary causes of death and disability worldwide, ${ }^{1}$ with a considerable proportion of those having a stroke developing significant persistent cognitive deficits that impact on functional ability. ${ }^{2}$ Cognitive impairment has been reported in over half of patients 6 months poststroke and is associated with increased disability and a poorer quality of life (QoL), ${ }^{3}$ while cognitive impairment in the acute phase poststroke is associated with depressive symptoms in the longer term. ${ }^{4}$ Those with moderate poststroke cognitive impairment are six times more likely to transition to incident dementia compared with those without cognitive impairment, ${ }^{5}$ with up to a quarter of patients with cognitive impairment diagnosed with dementia in the 3 years following stroke. ${ }^{6}$ Furthermore, it has been shown that $10 \%$ of patients develop dementia 
following a first-ever stroke, and over one-third develop dementia following a recurrent stroke. ${ }^{7}$ As such, there is a strong association between cognitive impairment and nursing home admission, particularly in those individuals affected by a more severe stroke. While the recovery of physical function poststroke has been the main focus of rehabilitation and research, with evidence demonstrating significant improvements following physical rehabilitation, ${ }^{89}$ rehabilitation of poststroke cognitive impairment has received considerably less attention. Despite the prevalence of cognitive impairment poststroke, and the associated implications for stroke survivors and burden on carers and the healthcare system, the efficacy of existing psychological interventions for the rehabilitation of cognitive impairment following stroke has yet to be established.

Cognitive rehabilitation has been defined as a 'systematic, functionally oriented service of therapeutic activities that is based on assessment and understanding of the patient's brain-behavioural deficits'. ${ }^{10}$ Five previous Cochrane reviews have been conducted in the area of poststroke cognitive rehabilitation. Specifically, these reviews have focused on occupational therapy for cognitive impairment, ${ }^{11}$ memory deficits, ${ }^{12}$ executive dysfunction, ${ }^{13}$ spatial neglect ${ }^{14}$ and attention deficits ${ }^{15}$ following stroke. Each has concluded that the effectiveness of cognitive rehabilitation aimed at each of these domains separately has yet to be established. However, the stringent nature of eligibility criteria for inclusion in these reviews could have resulted in the exclusion of important non-randomised controlled studies. The pattern of poststroke cognitive impairment suggests that deficits may be evident across all cognitive domains rather than being confined to one cognitive domain, ${ }^{16}{ }^{17}$ with lesion location predicting the severity of cognitive impairment across different cognitive domains following stroke. ${ }^{18} 19$ Despite the evidence suggesting more diffuse cognitive impairment poststroke rather than domain-specific deficits, there is, as yet, no review of psychological interventions for poststroke cognitive impairment that includes the full range of psychological interventions and that targets all forms of cognitive impairment (eg, including memory, attention, executive function and so on). While a Cochrane review of randomised controlled trial (RCTs) of psychological interventions for poststroke cognitive impairment is now planned by our group, ${ }^{20}$ this current review aims to capture those non-randomised controlled studies that do not meet the strict inclusion criteria of a Cochrane review but may be of value when designing a cognitive rehabilitation programme for poststroke cognitive impairment.

Therefore, the aims of the present systematic review are to identify which types of (non-randomised) psychological interventions have been employed to improve cognitive function poststroke and to assess the efficacy of these interventions in stroke survivors. The overarching goal is to inform the development of a cognitive rehabilitation intervention for individuals who experience cognitive impairment following stroke.

\section{METHODS AND ANALYSES}

\section{Study design}

This systematic review protocol will be reported in accordance with the Preferred Reporting Items for Systematic Review and Meta-Analysis Protocols (PRISMA-P). ${ }^{21}{ }^{22}$ Results will be reported in line with the Preferred Reporting Items for Systematic Reviews and Meta-Analyses statement. ${ }^{23} 24$

\section{Study registration}

In accordance with the PRISMA-P guidelines, this systematic review protocol was registered with the International Prospective Register of Systematic Reviews (PROSPERO) on 30 June 2017 (registration number: CRD42017069714; http://www.crd.york.ac.uk/PROSPERO/display_record. asp?ID=CRD42017069714)

\section{ELIGIBILITY CRITERIA \\ Types of study}

All non-randomised controlled studies and quasi-randomised controlled trials examining psychological interventions to improve cognitive function following stroke will be included in this systematic review, including feasibility studies, pilot studies, experimental studies and quasi-experimental studies. RCTs, review articles, letters, editorials, qualitative studies, case studies, animal studies and study protocols will be excluded.

\section{Participants}

Studies of an adult population (age 18+ years) will be included. Studies of participants with mixed aetiologies (eg, traumatic brain injury/stroke mix) will be excluded unless data are available, or made available on contacting the study authors, for those participants with a primary diagnosis of stroke (ischaemic, intracranial haemorrhagic and subarachnoid haemorrhage) or if the study has more than $75 \%$ of people with stroke in their sample. ${ }^{15}$

\section{Types of interventions}

Given the wide variation in types of interventions to address poststroke cognitive impairment, psychological interventions of any type and duration intended to rehabilitate cognition poststroke will be included. Examples of the eligible interventions will include: neuropsychological interventions; patient education interventions (video, books, leaflets, posters, videos and interactive modules); electronic interventions (eg, use of iPads and tablets); mobile phone apps including brain training apps/games; cognitive and/or behavioural interventions including problem solving; strategy training (eg, errorless learning, mnemonic strategies and vanishing cues); goal management training; and self-efficacy training. Studies with pharmacological or other non-psychological interventions will be excluded.

\section{Comparisons or control}

Studies addressing psychological interventions to improve cognition following stroke in comparison with a usual/ 
routine care control arm or an active control arm will be included.

\section{Outcome measures}

The outcome of interest is improved cognition after stroke, using a validated measure of domain-specific cognitive function, including those comprising the National Institute of Neurological Disorders and Stroke (NINDS) $30 \mathrm{~min}$ or $60 \mathrm{~min}$ battery of cognitive assessment. ${ }^{25}$ As a number of studies report scores from cognitive screening tools such as the Montreal Cognitive Assessment, ${ }^{26}$ Mini-Mental State Examination (MMSE) ${ }^{27}$ and Abbreviated Mental Test, ${ }^{28}$ these validated measures of cognition will also be acceptable. Other validated measures of domain-specific cognitive function are also acceptable, as are validated measures of subjective cognitive function (eg, Cognitive Failures Questionnaire ${ }^{29}$ and Metamemory in Adulthood Questionnaire ${ }^{30}$ ) and goal attainment scaling. ${ }^{31}$

Secondary outcomes of interest include reports of functional abilities in daily life and quality of life (QoL), including activities of daily living (ADL), for example, using the modified Rankin Scale ${ }^{32}$; instrumental activities of daily living, for example, using the Nottingham Extended Activities of Daily Living scale ${ }^{33}$; QoL, based on stroke specific or generic QoL assessment measures; and subsequent incidence of recurrent stroke, dementia, cardiovascular events or all-cause mortality.

\section{Search strategy for the identification of relevant studies}

The search strategy has been developed in collaboration with a subject librarian. Three databases covering the medical and psychological peer-reviewed literature will be searched: PubMed (http://www.ncbi.nlm.nih. gov/pubmed/), Embase (https://www.embase.com) and PsycINFO (http://www.apa.org/pubs/databases/ psycinfo/index.aspx). The PubMed search strategy is detailed in online supplementary appendix 1 . These terms will also be mapped to Medical Subject Headings terms and similar terms in Embase and PsycINFO, the search dating from the beginning of the index to February 2017. The search will be restricted to articles published in English.

Searches will be exported to EndNote X7 to build a master file of all references. In addition to the database searches, the reference list of included articles will be reviewed for relevant studies. A citation search will also be carried out to identify papers citing included articles, using Web of Science. A hand-search will also be conducted of the four journals that generate the greatest number of relevant articles.

\section{Screening of the studies}

Duplicates will be identified using EndNote X7's 'find duplicates' function. Titles and abstracts will be assessed for eligibility by one reviewer (NAM). Depending on the volume of papers generated by the search, at least a random $50 \%$ will be independently double-screened between four second reviewers (MEW, IJ, AG and DR). The full texts of papers identified as potentially eligible will be obtained for independent review by two reviewers. Any differences between reviewers will be resolved through discussion, with reference to a third independent reviewer $(\mathrm{AH})$ where necessary.

\section{Data extraction}

Data from included studies will be extracted using a standardised, prepiloted data extraction form. Two reviewers will extract data independently, with discrepancies identified and resolved through discussion, including with a third author where necessary. Extracted information will include: authors, study design, sample size (baseline and follow-up), sample description, target population characteristics, intervention type, intervention content, control (placebo and no treatment), length of follow-up, type of outcome, primary and secondary outcomes (listed above), comments and study conclusions. Study authors will be contacted for missing data or further information if necessary.

\section{Risk of bias}

Two authors will assess the strengths and weaknesses of each eligible study using the Risk of Bias in Non-randomized Studies of Interventions tool by the Cochrane Collaboration. ${ }^{34}$

No study will be excluded as a result of findings from the risk of bias assessments. However, if substantial variation in risk of bias of included studies is found, results will be synthesised separately for studies at high risk and low risk of bias.

\section{Quality of evidence}

The quality of the evidence of the studies will be assessed by two reviewers (NAM and MEW) based on the Grading of Recommendations Assessment, Development and Evaluation. ${ }^{35}$ The quality of the studies will be judged as high (further research is very unlikely to change the confidence in the effect estimates), moderate (further research is likely to have an important impact on the confidence in the effect and may change the estimate), low (further research is very likely to have an important impact on the confidence in the effect and is likely to change the estimate) and very low (any estimate of the effect is very uncertain). ${ }^{35}$

\section{Strategy for data synthesis}

Meta-analysis will be conducted provided that the studies/ methods are sufficiently homogeneous regarding the interventions and outcomes and, if sufficient data are available, to synthesise the direction, size and consistency of the possible effects using Stata V.14. Where there are no established thresholds for meaningful change for a given measure, the effect size (ES) thresholds suggested by Cohen $^{36}$ will be used: 'trivial' (ES <0.20), 'small' (ES $\geq 0.20<0.50$ ), 'moderate' ( $\mathrm{ES} \geq 0.50<0.80$ ) or large (ES $\geq 0.80$ ). Where necessary and possible, effect sizes will be adjusted to account for the correlation between 
baseline and outcome measures, as outlined by Middel and van Sonderen. ${ }^{37}$ If meta-analysis is not possible due to substantial heterogeneity, and so on, a narrative synthesis of the findings from the included studies will be provided, structured around the type of intervention, target population characteristics, type of outcome and intervention content. Heterogeneity will be quantified using the $\mathrm{I}^{2}$ statistic.

\section{Analyses of subgroup or subsets}

If sufficient data are available, subgroup analyses will be conducted. These analyses will assess differences between age of participants with stroke $(<65 \mathrm{vs} \geq 65)$; impact of depression and/or fatigue on cognitive performance; objective versus subjective improvement in cognition; type of intervention (eg, self-efficacy training vs education vs electronic, brief vs intensive, group vs individual and brief healthcare professional (HCP) contact vs longer term HCP contact and so on); duration and delivery of intervention; timing of outcome measures (eg, direct vs late effects of the intervention); and quality and risk of bias.

\section{DISCUSSION}

To the best of our knowledge, this review will be the first to investigate non-randomised controlled studies of the effectiveness of psychological interventions aimed at improving general cognitive function poststroke. Previous reviews have examined domain-specific interventions and outcomes such as attention, memory, executive function and spatial neglect, with each review concluding that effectiveness of cognitive rehabilitation aimed at each of these domains separately has yet to be established. ${ }^{12-15}$ However, the pattern of poststroke cognitive impairment typically is diffuse in nature, affecting a number of cognitive domains. ${ }^{16}{ }^{17}$ Furthermore, due to the stringent eligibility criteria of previous reviews, important studies may not have been included. These factors may limit the interpretation of the findings regarding the impact of interventions aimed at improving cognitive function in stroke survivors. Considering that cognitive impairment is a risk factor for progression to dementia, particularly in the context of further stroke, ${ }^{7}$ it is important to investigate the effectiveness of different types of psychological interventions to improve cognitive function in those with poststroke cognitive impairment.

The results of this review will provide evidence regarding which types, duration and delivery of psychological interventions are effective for managing poststroke cognitive impairment and will, in turn, inform the development of a cognitive rehabilitation programme as part of a wider study, the StrokeCog study, ${ }^{38}$ aimed at improving cognitive function poststroke. Furthermore, if sufficiently homogenous data are available to conduct a meta-analysis, HCPs will have information available regarding the expected effect size associated with a given intervention. This information will be useful for planning of rehabilitation services for those with poststroke cognitive impairment.

\section{Protocol amendments}

Protocol amendments will be documented with the date of each amendment and with a description of the change and the rationale.

\section{Author affiliations}

${ }^{1}$ Division of Population Health Sciences, Royal College of Surgeons in Ireland, Dublin, Ireland

${ }^{2}$ Economic and Social Research Institute, Dublin, Ireland

${ }^{3}$ Royal College of Surgeons in Ireland Library, Dublin, Ireland

${ }^{4}$ School of Physiotherapy, Royal College of Surgeons in Ireland, Dublin, Ireland

${ }^{5}$ Department of Psychology, Beaumont Hospital, Dublin, Ireland

${ }^{6}$ Department of Geriatric and Stroke Medicine, Royal College of Surgeons in Ireland, Dublin, Ireland

${ }^{7}$ Department of Geriatric and Stroke Medicine, Beaumont Hospital, Dublin, Ireland

Contributors All authors contributed to the conception and design of the study, the development of the search strategy, the establishment of the inclusion and exclusion criteria, data extraction criteria, analyses and interpretation. NAM, DR, IJ, $A G$ and MEW will perform the study search, screening and extraction of data. NAM drafted the manuscript, and AH, KEB, DW, NP, FH, and FD provided critical revision of the paper. All authors read and approved the final manuscript.

Funding This research was funded by the Health Research Board of Ireland Interdisciplinary Capacity Enhancement (ICE) award (2016-2019): The StrokeCog study: modelling and modifying the consequences of stroke-related cognitive impairment through intervention (Grant code: ICE-2015-1048) and HRB RL-151579 awarded to KEB.

Competing interests None declared.

Provenance and peer review Not commissioned; externally peer reviewed.

Open Access This is an Open Access article distributed in accordance with the Creative Commons Attribution Non Commercial (CC BY-NC 4.0) license, which permits others to distribute, remix, adapt, build upon this work non-commercially, and license their derivative works on different terms, provided the original work is properly cited and the use is non-commercial. See: http://creativecommons.org/ licenses/by-nc/4.0/

(C) Article author(s) (or their employer(s) unless otherwise stated in the text of the article) 2018. All rights reserved. No commercial use is permitted unless otherwise expressly granted.

\section{REFERENCES}

1. Feigin VL, Norrving B, Mensah GA. Global burden of stroke. Circ Res 2017;120:439-48.

2. Tatemichi TK, Desmond DW, Stern Y, et al. Cognitive impairment after stroke: frequency, patterns, and relationship to functional abilities. J Neurol Neurosurg Psychiatry 1994;57:202-7.

3. Mellon L, Brewer L, Hall P, et al. Cognitive impairment six months after ischaemic stroke: a profile from the ASPIRE-S study. BMC Neurol 2015;15:1-9.

4. Nys GM, van Zandvoort MJ, van der Worp HB, et al. Early cognitive impairment predicts long-term depressive symptoms and quality of life after stroke. J Neurol Sci 2006;247:149-56.

5. Narasimhalu K, Ang S, De Silva DA, et al. Severity of CIND and $\mathrm{MCl}$ predict incidence of dementia in an ischemic stroke cohort. Neurology 2009;73:1866-72.

6. Sachdev PS, Chen X, Brodaty $\mathrm{H}$, et al. The determinants and longitudinal course of post-stroke mild cognitive impairment. $J$ Int Neuropsychol Soc 2009;15:915-23.

7. Pendlebury ST, Rothwell PM. Prevalence, incidence, and factors associated with pre-stroke and post-stroke dementia: a systematic review and meta-analysis. Lancet Neurol 2009;8:1006-18.

8. Horgan F, Hickey A, McGee H, et al. National audit of stroke care. Dublin, Ireland, 2008. http://epubs.rcsi.ie/psycholrep/17/

9. Saka O, McGuire A, Wolfe C. Cost of stroke in the United Kingdom. Age Ageing 2009;38:27-32.

10. Cicerone KD, Dahlberg C, Malec JF, et al. Evidence-based cognitive rehabilitation: updated review of the literature from 1998 through 2002. Arch Phys Med Rehabil 2005;86:1681-92.

11. Hoffmann T, Bennett S, Koh CL, et al. Occupational therapy for cognitive impairment in stroke patients. Cochrane Database Syst Rev 2010;9:CD006430. 
12. das Nair R, Cogger $\mathrm{H}$, Worthington $\mathrm{E}$, et al. Cognitive rehabilitation for memory deficits after stroke. Cochrane Database Syst Rev 2016;9:CD002293.

13. Chung CSY, Pollock A, Campbell T, et al. Cognitive rehabilitation for executive dysfunction in patients with stroke or other adult nonprogressive acquired brain damage. Cochrane Database Syst Rev 2013;4:CD008391.

14. Bowen A, Hazelton C, Pollock A, et al. Cognitive rehabilitation for spatial neglect following stroke. Cochrane Database Syst Rev 2013;7:CD003586.

15. Loetscher T, Lincoln NB. Cognitive rehabilitation for attention deficits following stroke. Cochrane Database Syst Rev 2013;5:CD002842.

16. Sachdev PS, Brodaty $H$, Valenzuela MJ, et al. The neuropsychological profile of vascular cognitive impairment in stroke and TIA patients. Neurology 2004;62:912-9.

17. Vasquez BP, Zakzanis KK. The neuropsychological profile of vascular cognitive impairment not demented: a meta-analysis. J Neuropsychol 2015;9:109-36.

18. Barker-Collo S, Starkey N, Lawes CM, et al. Neuropsychological profiles of 5-year ischemic stroke survivors by Oxfordshire stroke classification and hemisphere of lesion. Stroke 2012;43:50-5.

19. Nys GM, Van Zandvoort MJ, De Kort PL, et al. Domain-specific cognitive recovery after first-ever stroke: a follow-up study of 111 cases. J Int Neuropsychol Soc 2005;11:795-806.

20. Hickey A, Merriman NA, McCabe G, et al. Psychological interventions for managing cognitive impairment after ischaemic stroke. http://www.cochrane.org/title/psychological-interventionsmanaging-cognitive-impairment-after-ischaemic-stroke

21. Moher D, Shamseer L, Clarke M, et al. Preferred reporting items for systematic review and meta-analysis protocols (PRISMA-P) 2015 statement. Syst Rev 2015;4:1.

22. Shamseer L, Moher D, Clarke M, et al. Preferred reporting items for systematic review and meta-analysis protocols (PRISMA-P) 2015: elaboration and explanation. BMJ 2016;354:i4086.

23. Moher D, Liberati A, Tetzlaff J, et al. Preferred reporting items for systematic reviews and meta-analyses: the PRISMA statement. Ann Intern Med 2009;151:264-9.

24. Liberati A, Altman DG, Tetzlaff J, et al. The PRISMA statement for reporting systematic reviews and meta-analyses of studies that evaluate health care interventions: explanation and elaboration. PLoS Med 2009;6:e1000100.
25. Hachinski V, ladecola C, Petersen RC, et al. National Institute of Neurological Disorders and Stroke-Canadian Stroke Network vascular cognitive impairment harmonization standards. Stroke 2006;37:2220-41.

26. Nasreddine ZS, Phillips NA, Bédirian V, et al. The Montreal Cognitive Assessment, MoCA: a brief screening tool for mild cognitive impairment. J Am Geriatr Soc 2005;53:695-9.

27. Folstein MF, Folstein SE, McHugh PR. "Mini-mental state". A practical method for grading the cognitive state of patients for the clinician. J Psychiatr Res 1975;12:189-98.

28. Hodkinson HM. Evaluation of a mental test score for assessment of mental impairment in the elderly. Age Ageing 1972;1:233-8.

29. Broadbent DE, Cooper PF, FitzGerald P, et al. The Cognitive Failures Questionnaire (CFQ) and its correlates. Br J Clin Psychol 1982;21(Pt 1):1-16.

30. Dixon RA, Hultsch DF, Hertzog C. The Metamemory in Adulthood (MIA) questionnaire. Psychopharmacol Bull 1989;24:671-88.

31. Kiresuk TJ, Sherman RE. Goal attainment scaling: A general method for evaluating comprehensive community mental health programs. Community Ment Health J 1968;4:443-53.

32. Sulter G, Steen C, De Keyser J. Use of the Barthel index and modified Rankin scale in acute stroke trials. Stroke 1999;30:1538-41.

33. Nouri FM, Lincoln NB. An extended activities of daily living scale for stroke patients. Clin Rehabil 1987;1:301-5.

34. Sterne JA, Hernán MA, Reeves BC, et al. ROBINS-I: a tool for assessing risk of bias in non-randomised studies of interventions. BMJ 2016;355:i4919.

35. Guyatt GH, Oxman AD, Vist GE, et al. GRADE: an emerging consensus on rating quality of evidence and strength of recommendations. BMJ 2008;336:924-6.

36. Cohen J. Statistical power analysis for the behavioural sciences. rev. ed. New York, NY: Academic Press, 1977.

37. Middel B, van Sonderen E. Statistical significant change versus relevant or important change in (quasi) experimental design: some conceptual and methodological problems in estimating magnitude of intervention-related change in health services research. Int $\mathrm{J}$ Integr Care 2002;2:e15.

38. Hickey A, Bennett K, Wren M-A, et al. The StrokeCog study: modelling and modifying the consequences of stroke-related cognitive impairment through intervention. http://www.hrb.ie/ research-strategy-funding/grants-and-fellowships/funding-awarded/ funding-award/awards//639/. 